\title{
Current Situation of Environment Protection Sizing Agent and Paste
}

\author{
Hui Xiao \\ School of Textiles, Tianjin Polytechnic University, Tianjin 300160, China \\ E-mail: xiaohui2135267@163.com \\ Wei Zhang (Corresponding author) \\ Textlie and Garmengt College, Hebei University of Science and Technology \\ Shijiazhuang 050018, China \\ E-mail: zhangwei2999vivian@126.com
}

\begin{abstract}
From the angel of environment protection, through analysing the properties of three majority types of sizing agent, such as starch(modified starch), PVA(polyvinyl alcohol) and acrylic size, we attempted to compare the sizing performance indices of several different environment protection sizing agents. We also briefly introduced several printing pastes with research values currently which could substitute sodium alginate.
\end{abstract}

Keywords: Environment protection sizing agent, Paste, Performance, Evaluation

\section{Introduction}

Textile sizing agents were classified into three main types, viz. Modified starch, PVA and acrylic size currently, which had an important status in the market of home and abroad, especially for starch size, accounting for about $70 \%$ total size consumption. In the international market, consumption of PVA is equivalent to acrylic size, however, in the domestic market, consumption of PVA is about two times of acrylic size. PVA belongs to refractory biodegradable organic substances, and could cause serious pollution to environment. Aimed at those problems, such as large consumption of PVA in textile size in China, environmental pollution and so on, which could affect the textile exports in China, researches on green size instead of PVA have been developed in China in recent years. Green size is a size that accord with environment protection, not only in line with functional requirements of warp sizing, but also attenuating the pollution to water and air imposed by sizing to the lowest level, guaranteeing biodegradable desizing wastewater with less pollution, and reducing the toxic substances remaining in the textile to the level within regulated limitation.

\section{Research progress of environment protection sizing agent}

\subsection{Modification of existed sizing agent instead of PVA}

At present, existed biodegradable materials, such as modified starch, acrylate, polyester and so on, have been applied to prepare green size, with attempts to substitute PVA partly or totally, which is a main approach to explore size green by many researchers.

\subsubsection{Modified starch}

Modified starch is the main size used for yarn sizing of natural fiber such as all cotton, for ameliorating the compatibility between starch and synthetic fiber, and flexibility of size film after modification, and could be used in the sizing process of blended yarn such as polyester/cotton instead of PVA. Modified starch size that is used frequently at home or abroad at present is starch derivatives. Researches about grafted starch have been developed to a certainty.

Currently, starch derivatives have been the main green size due to its compatibility to synthetic fiber and flexibility of size film, as well as good stability and dispersivity of viscosity. When acetyl and hydrophobic ester group were induced into acetate starch, its adhesion to natural and synthetic fiber were enhanced. Not only sizing wearability elevated, which made fuzzy yarns more comfortable, due to the induction of ester group, film forming ability also increased, which made the formed film more flexible and ameliorated the strength elongation property of sizing yarn. Currently, acetate starch could substitute 20 30\% PVA size in China. In order to substitute more PVA, more attention has been paid to grafted starch by researchers. Grafted starch was prepared to be used in the sizing process of polyester/cotton blended yarn, and could substitute PVA in large scale. If graft technology was taken further amelioration, grafted starch would be applied in large proportion, and even totally used instead of PVA. Polymethacrylic acid grafted starch prepared by Mostafa was obtained by grafting methacrylic acid to corn starch in potassium permanganate/ citric acid 
system and performance of such grafted starch was greatly enhanced. Fracture strength, breaking tensile rate and wearability of such sizing yarn after cotton textile sizing were all excellent. although there was a certain difference in size performance from PVA, it could be singly used in sizing process instead of PVA. Zhang et al had prepared a grafted starch size with well permeability to fiber by grafting polyacrylic acid. Such size possessed good sizing performance, size strength and tensile rate, comfortable fuzzy yarns, and could be used in sizing process of polyester/cotton completely instead of PVA. However, how to substitute in other textile totally should still need further investigation(Qin, 2006, PP. 5-6).

\subsubsection{Acrylic sizing agent}

Acrylic sizing agent is the general name of homopolymer, copolymer or their combination of acrylic monomers. Synthetic size with the main body of acrylic ester is the paramount kind of acrylic sizing agents, and has excellent adhesion to hydrophobic synthetic fiber, tender size film with lower strength, large deformability and moisture absorption stickiness viscosity. When sizing, it was favorable to reduce size fuzzy yarns and increase wearability. Due to the flaw of PVA size in environment protection, investigating and developing acrylic sizing agents has been imperative at present. Acrylic sizing agents includes three main types: poly acrylate, polyacrylamide and polymethyl acrylate. Poly acrylate type has excellent adhesion to hydrophilic fiber, large moisture absorption and heavy stickiness viscosity, and would have well sizing performance in combination with low proportion modified starch. PAAM (polyacrylamide) type has large moisture absorption and heavy stickiness viscosity, and good adhesion to hydrophilic fiber, with $25 \%$ of solid-containing content. It is a kind of monomer homopolymer, and its quality is easy to control. It could be made to solid formulation. Acrylate type is the high technological product of acrylic acids, and a copolymer of acrylate monomers, with two or three monomers. It has good adhesion to hydrophobic fiber, and modified moisture absorption stickiness viscosity compared to the previous two types.

Performance and quality of liquid or solid state acrylic size made in China is identical compared to foreign products. It is possible that combination of acrylic size and modified starch was applied in the sizing process of thin cotton fabric completely instead of PVA. Acrylic size has good film forming ability and adhesion, and excellent size film strength, flexibility and wearability, which is the cardinal basic condition of less or no consumption of PVA in China(Qin, 2006, PP. 5-6).

New type acrylic size STSX-1 developed by Donghua University was prepared by copolymerization of hydrophilic monomers, i.e. acrylamide and acrylic acid, and hydrophobic monomers, i.e. butyl acrylate and vinyl acetate, with attempts to obtain higher adhesion to polyester/cotton in favor of elevating size film strength, flexibility and fuzzy yarns comfort. Size film had lower fracture strength and lower leasing resistance. Moisture absorption of its size film is similar to PVA, with no thickness viscosity, lower cost and good stability. Consumption of PVA in the size formula could be reduced from $55 \%$ to $25 \%$ by using STSX-1, and less environmental pollution was imposed. Studies on acrylic ester sizing agents was also one of the focuses abroad. Hiroshi Y in Japan had used vinyl acetate copolymer heat melt sizing agents in the sizing process of polyester and cotton yarns and attained excellent effects(Zhang, 2007, PP. 15-16).

\subsubsection{Polyester sizing agent}

Hydrophilic polyester sizing agent is a new type textile size which is a macromolecular structure with ester group (-COO-) and hydrophilic groups, synthesized by condensation and polymerization using synthetic polyester resins or similar materials such as TPA(terephthalic acid), EG(ethylene glycol) and so on. For macromolecular of polyester contained benzene ring and ester group, similar to the molecular structure of polyester fiber, it had excellent adhesion to polyester fiber, and had good water solubility due to the presence of strong hydrophilic groups. Ester groups in such molecule were susceptible to hydrolyze in the alkali medium, and thus had excellent desizing performance. It could ameliorate the desizing performance of starch and PVA; Viscosity and surface tension of such size were all low, and had good wettability and permeability to yarns. Currently, it was mainly applied in the sizing process of polyester filament, but as far as polyester staple fiber or polyester and cotton blend was concerned, people had different opinions( $\mathrm{Qu}, 2005$, PP. 257-260).

\subsection{Other green environment protection sizing agent}

Another approach to size green was to utilize new type material. Existing sizing agents have been modified to substitute PVA at present, and most of them were far from satisfactory due to their wearability and breaking strength. Therefore, attempts to developing sizing agents to remedy and substitute the defects of PVA by using new type materials, such as nanometer material, have been attracted many researchers' attention.

\subsubsection{Inorganic nanometer material}

Inorganic nanometer size is virtually a kind of size additive, and its production methods have three kinds as follows: One is to produce in the manner of dispersive way by using nano-scale human titanium oxide or silicon dioxide; Two is to produce directly by using origin materials such as titanium tetraoxide, egtazic acid ester, silicon powder and so on; Three is to blend starch and nanometer material to form solid nano-scale size additive. Nanometer sizing agents 
prepared by different materials and methods had great difference in performance: solid amount had three types such as $15 \%, 30 \%$ and $40 \% ; \mathrm{pH}$ value had two scopes such as $2 \sim 4$ and 7 8.5; Particle diameter had also two scopes such as $100 \sim 200 \mathrm{~mm}$ and $50 \sim 80 \mathrm{~mm}$. In the sizing process of medium-fine total cotton textile, it could mostly take the place of "impure size PVA" ; In the sizing process of polyester-cotton fabrics, it could partly substitute PVA. And thus it had significant economic and social benefits(Yang, 2008, PP. 5-6).

\subsubsection{Natural plant gum sizing agent}

Main variety guar gum is a kind of natural macromolecular plant gum with excellent water solubility, and extracted from the endosperm of guar beans planted in India and Pakistan. It has excellent adhesion to both total cotton and polyester-cotton. In combination with starch and $\mathrm{CMC}$, its size film had weaker fracture strength, but breaking tensile rate attained $18.59 \%$ with great wearability and 1043 times buckling resistance(Yang, 2008, PP. 5-6).

\subsection{Existing problems and development of green sizing agent}

Although people have developed textile size partly or totally instead of PVA, some problems still existed in their researches, for example, starch derivatives applied at present could not completely substitute PVA. Studies on modified starch should focus on developing grafted starch with excellent performance, but production process of grafted starch was complicated with high cost and low market share. Acrylic sizing agents still had the problems of re-viscosity after moisture absorption, and were also expensive. Therefore, studies on acrylic sizing agents should utilize the designable characteristic of copolymer size and solve the existing problems though optimizing their ratio. Solid acrylic sizing agents were easy to use with low transport cost, so developing such size would be one of the developing trends. Moreover, due to good film-forming property of PVA, excellence of size film performance could not be totally substituted. How to seek a proper approach to solve the refractory problems of PVA would also be of great significance. Studies on such area focused on two aspects currently as follows, one was the modification of PVA, with attempts to make it possess the property of biodegradation. Two was to develop strains which could degrade PVA. Only with unremitting efforts, amid at higher quality, more function, less compositions, seriation, less or no consumption of PVA, or developing biodegradable PVA, real green textile size of practical values could be developed.

\section{Research progress of environment protection pastes}

Printing pastes are important part of printing color sizing, and are close associated with printing operation performance, definition of pattern contour, hand feeling and so on. Sodium alginate has long been main printing pastes with excellent performance long utilized by human being. With the development of printing technology, higher requirements for printing pastes were imposed, and single application of such pastes were far from the requirements of printing with high quality, with the defects of undesirable rheology and printing effects of round(flat) screen printing, especially for high mesh screen printing, fine design and large-scale printing. Moreover, sodium alginate has been transformed into edible food gradually in recent years, and its price increased, which resulted in the increasing of printing production cost. Consequently, people were urgent to seek a cheap printing pastes with excellent performance instead of sodium alginate.

Guar gum was treated by hydrogen peroxide, and hydroxyls in its molecule were oxidized into aldehyde or carboxyl groups, which was applied in producing modified guar gun. When guar gum or sodium alginate treated by hydrogen peroxide was applied in active printing, K/S value resulted from oxidizing guar gum was far higher than that from sodium alginate; Dry and wet rubbing fastness was not lower; But winding length of printing fabrics was larger, namely rigid hand feeling. With the increasing of hydrogen peroxide consumption, all indices of printing fabrics were ameliorated(Huang, 2008, PP. 5-8).

PA-CMS is a kind of crosslinked carboxymethyl starch. Experiments indicated that it possessed excellent printing performance and well comprehensive printing effects, and could be applied as printing pastes for active dyes or disperse-active dyes. It was a desirable substitute for sodium alginate(Ma, 2000, PP. 267-269).

Bentonite is a kind of inorganic minerals, and after a serial treatments of impurity removal, modification, expansion, it would be a refined printing pastes. With the appearance of white granule, when added water, it was milky white after agitation. Such paste was homogeneous and exquisite, and was resistant to acid and alkali, as well as additives common used in general printings. It had good stability, and certain viscosity and consistency, but when water addition attained a certain level, its viscosity would drop abruptly, and thus caused the poor water retention property. Such paste had poor fluidity in normal state, when given force, its viscosity decreased. As seen from rheological curve, it belonged to typical pseudoplastic fluid. Due to poor fluidity and water retention property, when single used as printing paste, it would cause bleeding and uneven pulp fluid, but in combination with certain proportion seaweed, its rheological property would be greatly modified(Yun, 1999, PP. 25-26).

The most significant advantages of bentonite as printing paste is no binding force with dyes and fibers with high color yield after printing. Paste is easy to wash, and resultant wastewater cause no pollution to environment. Disadvantages are as follows: (1) poor permeability; (2) low adhesive force; (3) poor water retention property, and weak dilution 
resistance. Therefore, it was tough to attain good printing effect using single bentonite, and it should be blended with other pastes to be compound paste. Above mentioned defects would be overcome by blending which could also protect bentonite, and enhance its stability to electrolytes. After bentonite blended with sodium alginate in a proper proportion, printing products possessed rich color yield, and its permeability and color fastness index attained the level when using sodium alginate; Blending size could lower the cost, attenuate the burden of wastewater treatment; Furthermore, it had great storage stability, and was hard to deteriorate. However, if in an improper proportion, phenomena of paste pressing and net destroying would occur, which need further investigation(Huang, 2008, PP. 5-8).

Synthetic thickener is a kind of synthetic macromolecule compounds, and monomers used are mainly acrylic acids, maleic acid and less crosslinked monomers. Used for thickener, it had good rheology, low forming paste rate and low solid mass percent of color paste. No hydroxyl but lots of carboxyls existed in molecule, which facilitated they had quite high negative charge density in alkali condition, and offered possibility for the printing of active dyes.

Synthetic paste $\mathrm{C}$, using polysaccharides of cotton linter as main original paste, is a new type anionic printing paste through reactions of crosslinking, etherifying, carboxylation and so on. For general printing fabrics, apparent color strength was used to evaluate colorizing effect of dyes. Through electronic color measurement of printing samples, K/S values in the surface were observed, percent of $\mathrm{K} / \mathrm{S}$ value all used sodium alginate as standard. Apparent color strength of synthetic paste $\mathrm{C}$ was higher than that of sodium alginate, with the increasing of original paste concentration, viscosity of synthetic paste $\mathrm{C}$ attained double increase compared to sodium alginate. For its good storage stability, resistant to all kinds of chemicals, it could be used in weak alkali, strong alkali or acid conditions, and suitable for many printing processes. Therefore, it was a kind of multifunctional printing paste(Wang, 2002, PP. 72-73).

\section{References}

Huang, Y., Zhu, P., \& Wang, B., et al. (2008). The development of reactive printing pastes. Textile Auxiliaries, 25(5):5-8.

Ma, C.H. (2000). The study and application of new type printpaste. Henan Science, 18(3):267-269.

Qin, Z.J. (2006). Development and application of green sizing agent. Progress in Textile Science \& Technology, 4:5-6.

$\mathrm{Qu}$, C.X. (2005). Application analyses on environment protection sizing agent. Cotton Textile Technology, 33(5):257-260.

Wang, H., Zheng, Y.D., \& Ren, X.Z. (2002). Synthesis Gum C Instead of Sodium Alginate in Reactive Printing. Journal of Zhongyuan Institute of Technology, 13(2):72-73.

Yang, Z.Q. (2008). Green environment protection textile size. Textile Decoration Science \& Technology, 1:5-6.

Yun, J.R., \& Zhou, L.Q. (1999). Application of Bentonite Paste in Printing with Reactive Dyes. Textile Dyeing and Finishing Journal, 21:25-26.

Zhang, C., Yu, S.M., \& Gao, X.L. (2007). Research progress of green textile size at home and abroad. Progress in Textile Science \& Technology, 6:15-16. 